\title{
A Game-Theoretic Analysis of Hezong and Lianheng-Simultaneous and Sequential Moves
}

\author{
Jue-Shyan Wang (Corresponding author) \\ Department of Public Finance, National Chengchi University \\ 64, Tz-nan Road, Sec. 2, Wenshan, Taipei 11623, Taiwan \\ E-mail: jswang@nccu.edu.tw \\ Sih-Ting Nian \\ Department of Public Finance, National Chengchi University \\ 64, Tz-nan Road, Sec. 2, Wenshan, Taipei 11623, Taiwan \\ E-mail: 96255025@nccu.edu.tw
}

The research is financed by National Science Council, Taiwan (NSC98-2410-H-004-042).

\begin{abstract}
This paper will study a three-player game with infinite periods where each player decides whether to league together to increase his winning rate. We discuss which one will be chosen to be the member of leagues. The results show that Hezong and Lianheng both exist in mixed strategy Nash equilibriums of simultaneous move game. It is possible that the fall of Jin and the Battle of Red Cliffs in Chinese history were just coincident or random events. Furthermore the players usually have a tendency to be in league with the strongest.
\end{abstract}

Keywords: Alliance, Coalition, League, Hezong, Lianheng, Three players

\section{Introduction}

The Warring States Period of Chinese history was a period when regional warlords absorbed smaller states around them. And seven major states, the Han, the Zhao, the Wei, the Qi, the Chu, the Yan and the Qin, were rising to prominence. These states were the famous Seven Warring States. At the end of the Warring States Period, Qin became disproportionately mighty compared to the other six states. In order to deal with the Qin threat, the six states had to figure out some strategies, such as Hezong or Lianheng. Hezong is to alliance among weaker power to repulse the strongest, while Lianheng is to unite with the strongest to participate in his domination.

As one of the weaker states, Su Qin advocated Hezong strategy to unite among six states so as to compete with the strongest Qin. On the other hand, to rival Su Qin's strategy, The Prime Minister of Qin, Zhang Yi, advocated Lianheng strategy. Although there were some initial triumphs in Hezong, Qin beat the six states one by one later. In addition, there were still some other examples of Hezong and Lianheng in history. The league of Sun Quan and Liu Bei to resist Cao Cao in the Three Kingdoms Period was an example of Hezong. The alliance of Germany and Soviet Union to invade Poland in World War II or the connection made between Mongol and Southern Song to attack Jin were examples of Lianheng.

\section{Insert Figure 1 Here}

For the sake of understanding the above strategy coalitions, we design a three-player model where the players contend for war trophies. Each player decides whether to league together to increase his winning rate. There is some literature to study this issue. Skaperdas (1996) suggests that tournaments, conflict, and rent-seeking have been modeled as contests in which all participants exert efforts to increase their own possibility of winning a prize. Two frequently used functional types are also axiomatized: the first one is that the winning possibility depends on the ratio of each player's effort, and the other one is that the winning possibility depends on the difference in efforts. 
Beest et al. (2003) assume that players in a coalition game not only pay attention to maximizing their own payoff but that they also ponder the harm that is caused to an excluded player by forming a small coalition. People are less willing to exclude others when it's explicitly harmful to exclude. And Beest et al. (2005) show that participants are more unwilling to exclude others in order to minimize their harm than to maximize their gains. And participants are most influenced by payoff valence when they are inclined to consider the viewpoint of others.

Beest et al. (2004a) show that when the alliance payoff is such that members of each possible alliance could obtain an equitable payoff share, the outcome tends to be alliances that also maximize the payoff of its members. Beest et al. (2004b) indicate that the payoff of alliance members is related to the resources they contribute to the alliance, and to the number of alternative alliances they can form. It is shown that distinction in alternatives leads to longer bargaining.

Bottom et al. (2000) test how negotiators' risk preferences affect the formation of coalitions. With inexperienced bargainers, risk preferences forecast coalition composition but not payoff distribution. For experienced bargainers, risk preferences are irrelevant to coalition membership but do forecast the distribution of payoffs. And Ikonnikova (2007) offers a new methodology to study how upstream and downstream players form leagues, bargain over joint profit sharing and invest. Profit of each league depends on the cooperation among outside players. Payoffs reflect the bargaining power and depend on capabilities of players.

Skaperdas (1998) examines the three-person/one-cake problem that a coalition between two players versus the third one will develop if and only if the Contest Success Function has an increasing returns character. While a coalition forms, there is an inclination to be formed by the weaker players against the strongest player. And Konrad (2008) indicates that the formation of a coalition in conflict situations is known to suffer from a corporate action problem and from the latent of internal conflict. They show that budget constraints of a middle size can overcome this strong disadvantage and interpret the formation of coalitions.

Whether information is complete or not, it also influences the formation of leagues. Sorokin (1994) studies the relationship between coalition formation and general deterrence in regional competition. General deterrence succeeds under complete information unless the latent attacker is stronger than the target and values the stakes highly, and the latent ally would never intervene. Besides, general deterrence under incomplete information fails at times because of the latent attacker's mistaken beliefs about the possibility that the target would win or the latent ally would intervene.

Furthermore, timing is also important. Esteban and Sákovics (2003) analyze a model of conflict with endogenous selection of effort, where subsets of the opponents may force the resolution to be sequential. First the league fights it out with the rest and later they fight it out through themselves. Ridder and Rusinowska (2008) also confirm that coalition is formed simultaneously or members are added one by one is an important factor in multipart coalition formation. However, if ideal positions of firms are acceptable to all coalition partners, then the result of coalition formation does not depend on order. Moreover, it is shown that it can be adverse to be a first mover.

Coalitions are common in political voting. For example, we may be in favor of your subject to barter for your support of ours. As a result, both of our subjects will be approved. Feix et al. (2007) point out that if two or more players in a voting game have on a binary subject independent opinion, they may have interest to develop a single voting coalition giving an average gain of influence for all of them. And in the so-called asymptotic limit, power is relative to the number of votes. Power of a coalition is equal to the sum of member's power.

Eguia (2006) proves that there subsist stable endogenous voting bloc structure and in a congress with two parties he shows how the incentives to develop a bloc depend on the sorts of agents, the size of the parties, and the regulations the blocs use to aggregate their preference. Eguia (2007) also studies the advantages that an alliance of agents obtains by developing a voting bloc to pool their votes and cast them all together. They also determine whether individual agents prefer to join in or step out of the bloc, and they find the different optimal interior voting rules that aggregate preferences within the alliance.

Tan and Wang (1997) study endogenous alliance formation in an environment where continuing conflict exists. Players contend for an indivisible prize and they can combine their resource together to raise their winning rate through alliance formation. They analyze problems of temporary cooperation within heterogeneous individuals who are opponents in nature. The game goes on until one individual winner is left. The outcome of a three-player game is the weakest two players form an alliance and fight against the strongest.

Nevertheless, we suppose it is an infinite period game, a war is costly, and the survivor can get a fixed amount of 
profit in each period in our paper. We are looking forward to that players' behavior and equilibriums will differ from the previous models. Neary (1996) considers that costly conflict causes a region of strategy space in which it is not individually reasonable for players to fight. The possibilities that armed players will contend, and that the richer player is the attacker, are endogenously determined.

We first discuss the problem in a simultaneous move game where players take pure strategies or mixed strategies. Then we substitute real numbers to the model in the completely mixed strategy case. Further, we analyze that players move sequentially. Will players form a coalition? Which one will be chosen to be the member of alliances? Does the league belong to Hezong or Lianheng? And does it correspond to the history events? Those are what we are interested in. In addition, coalitions are applied not only to military alliances, but also to contests, R\&D tournaments and political elections. These cases can be analyzed in the similar way.

\section{The model}

We assume that there are three players, and the strength of player $i$ is $r_{i}, i \in\{1,2,3\}$, where $r_{1}>r_{2}>r_{3}$. A war seems to be brewing among the three players. Each of them can choose to be in league with one of the others. However, if their choices don't match, singleton league will happen. (Note 1) If a league wins, the players in the league will further contest. Each member of the winning league will get $a / 2$ in the first period. In the second period, the winner will get $a+\delta v$ and the loser gets $0 . v$ is defined as follows:

$$
v=\sum_{t=0}^{\infty} \delta^{t}(a+b)=\frac{a+b}{1-\delta}
$$

$\delta$ is discount rate or the expectation of that a game exists in the next period, where $\delta \in(0,1)$. b represents the difference between war and peace, where $b>0$.

To simplify the discussion, we define four states of leagues. Lianheng 1 indicates player 1 and 2 league together, and the payoff are $\left(a_{11}, a_{12}, a_{13}\right)$. Lianheng 2 indicates player 1 and 3 league together, and the payoff are $\left(a_{21}, a_{22}, a_{23}\right)$. Hezong indicates player 2 and 3 league together, and the payoff are $\left(a_{31}, a_{32}, a_{33}\right)$. Singleton indicates singleton league, and the payoff are $\left(a_{41}, a_{42}, a_{43}\right)$.

Suppose the winning rate is the strength of each league over total strength. (Note 2) For instance, in the state of Lianheng 1 , the winning rate of player 1 and 2 is $\left(r_{1}+r_{2}\right) /\left(r_{1}+r_{2}+r_{3}\right)$ in the first period, and is $r_{i} /\left(r_{1}+r_{2}\right), i \in\{1,2\}$ in the second period. But the winning rate of player 3 is $r_{3} /\left(r_{1}+r_{2}+r_{3}\right)$ in the first period. While in the state of Singleton, the winning rate of player $i$ is $r_{i} /\left(r_{1}+r_{2}+r_{3}\right), i \in\{1,2,3\}$ in the first period.

\section{Simultaneous move game}

First, we discuss the condition that players choose their strategies simultaneously and independently. Therefore neither of them observes the other's move before making his choice. Player 1 can select to be in league with player $2\left(\ell_{2}\right)$ or $3\left(\ell_{3}\right)$, and so forth, as it is shown in Fig. 2.

For a start, we discuss situations in pure strategy. (Note 4) The state of Lianheng 1 includes condition (1) and (5)

in Table 1. In condition (1), player 1 chooses $\ell_{2}$ because $a_{11} \geq a_{21}$, player 2 chooses $\ell_{1}$ if $a_{12} \geq a_{42}$, and 
this implies $r_{2} \leq[a /(a+2 \delta b)] r_{1}$, and player 3 chooses $\ell_{1}$ because $a_{13} \geq a_{13}$. In condition (5), player 1 won't choose $\ell_{2}$ because $a_{11}<a_{41}$, which implies $r_{1}>[a /(a+2 \delta b)] r_{2}$. Thus, condition (5) won't exist.

The state of Lianheng 2 includes condition (2) and (4). But in condition (2), player 1 won't choose $\ell_{3}$ because $a_{21}<a_{11}$, which implies $r_{3}<r_{2}$. In condition (4), player 1 won't choose $\ell_{3}$ because $a_{21}<a_{41}$, which implies $r_{1}>[a /(a+2 \delta b)] r_{3}$. Then, neither condition (2) nor condition (4) will exist.

The state of Hezong includes condition (7) and (8). But in condition (7), player 2 won't choose $\ell_{3}$ because $a_{32}<a_{12}$, which implies $r_{3}<r_{1}$. In condition (8), player 2 won't choose $\ell_{3}$ because $a_{32}<a_{42}$, which implies $r_{2}>[a /(a+2 \delta b)] r_{3}$. Then, neither condition (7) nor condition (8) will exist.

The state of Singleton includes condition (3) and (6). In condition (3), player 1 chooses $\ell_{2}$ because $a_{41} \geq a_{21}$, player 2 choose $\ell_{3}$ if $a_{42} \geq a_{12}$, which implies $r_{2} \geq[a /(a+2 \delta b)] r_{1}$, and player 3 chooses $\ell_{1}$ if $a_{43} \geq a_{33}$, which implies $r_{3} \geq[a /(a+2 \delta b)] r_{2}$. In condition (6), player 1 chooses $\ell_{3}$ because $a_{41} \geq a_{11}$, player 2 chooses $\ell_{1}$ because $a_{42} \geq a_{32}$, and player 3 chooses $\ell_{2}$ if $a_{43} \geq a_{23}$, which implies $r_{3} \geq[a /(a+2 \delta b)] r_{1}$. Therefore, both condition (3) and condition (6) will exist.

In sum, when three players all take pure strategy, condition (1) stands if $r_{2} \leq[a /(a+2 \delta b)] r_{1}$, condition (3) stands if $r_{2} \geq[a /(a+2 \delta b)] r_{1}$ and $r_{3} \geq[a /(a+2 \delta b)] r_{2}$, and condition (6) stands if $r_{3} \geq[a /(a+2 \delta b)] r_{1}$. It is possible that Lianheng 1 and Singleton exist, and it corresponds to Equilibrium 1, 2, and 3 in Table 1.

After that, we analyze this problem in mixed strategy. Let $s$ denote the probability that player 1 selects $\ell_{2}$, meaning that his mixed strategy is $(s, 1-s)$. Let $t$ denote the probability that player 2 selects $\ell_{1}$, meaning that his mixed strategy is $(t, 1-t)$. Likewise, let $u$ denote the probability that player 3 selects $\ell_{1}$, meaning that his mixed strategy is $(u, 1-u)$.

There may be one of them, two of them, or all of them take mixed strategy. Equilibrium 4 is that three players all pick mixed strategy, Equilibrium 5 and 6 are two of them select mixed strategy, and Equilibrium 7 is only one player takes mixed strategy.

Thus, when three players all choose their strategies simultaneously, we have the following seven Nash 
equilibriums in Table 1. In these equilibriums, Equilibrium 1, 2 and 3 are pure strategy Nash equilibriums, and the others are mixed strategy Nash equilibriums. And Equilibrium 1, 2 and 3 in Table 1 correspond to Condition (1), (3) and (6) in Fig. 2.

Proposition 1. When three players move simultaneously,

(1) only Lianheng 1 and Singleton exist in pure strategy Nash equilibriums.

(2) Lianheng 1, Lianheng 2, Hezong, and Singleton all exist in mixed strategy Nash equilibriums.

Let $r_{3}$ be horizontal axis and $r_{2}$ be vertical axis. By the restrictions in Table 1, we can form eleven regions, as it is shown in Fig. 3. Because $r_{2}>r_{3}$, we only discuss the regions above $r_{2}=r_{3}$. The scope of each region is shown in Table 2. In addition, equilibriums and states which correspond to the regions are shown in Table 3.

By Fig. 3 and Table 3, we know that it exists only pure strategy equilibriums in region $A_{3}, A_{9}$ and $A_{11}$. On the contrary, it exists mixed strategy equilibrium merely in region $A_{1}$. Player 1 and 2 league together in all regions except $A_{3}$. Player 1 is in league with player 3 in region $A_{1}, A_{2}, A_{4}, A_{5}, A_{6}$ and $A_{7}$. Player 2 and 3 league together in region $A_{1}, A_{2}, A_{4}, A_{5}, A_{6}, A_{7}$ and $A_{10}$. Singleton league exists in region $A_{1}$, $A_{2}, A_{3}, A_{4}, A_{5}, A_{6}, A_{7}, A_{8}$ and $A_{10}$. And four states all exist in region $A_{1}, A_{2}, A_{4}, A_{5}, A_{6}$ and $A_{7}$.

When $r_{1}$ and $r_{2}$ get closer as in region $A_{1}$, only Equilibrium 4 exists. If each player has positive probability on every action, then completely mixed strategy happens. And while the difference among three players' strength is not much as in region $A_{5}$, completely mixed strategy, pure strategy Equilibrium 2 and pure strategy Equilibrium 3 happen.

Besides, Lianheng 1 exists in region $A_{9}, A_{10}$ and $A_{11}$. It means that when $r_{2}$ and $r_{3}$ are weak and close but $r_{1}$ is disproportionately strong, player 2 would have a tendency to be in league with player 1 . And Equilibrium 1 exists in $A_{6}, A_{7}, A_{8}, A_{9}, A_{10}$ and $A_{11}$. That is to say, Lianheng 1 of pure strategy Nash Equilibriums would exist while $r_{2}$ is weaker.

Now we instance that three players all take mixed strategy, and we substitute real number to $s, t, u$, where $\delta=0.9$ and $a=1$, as it is shown in Table 4 .

After that, multiply the probability and add them together for each state. Then we can get the probability of four states in Table 5. And we discover that when the cost of the war is bigger, the probability of Singleton is higher but the probability of Lianheng is smaller. 
Then we analyze the probability of four states when $b=2$. From Fig. 4 , there is little change in Singleton, so we ignore it and discuss the other three states in the following.

(1) From $(4,3,2)$ to $(5,3,2)$ :

When $r_{2}$ and $r_{3}$ are constant and $r_{1}$ raises from 4 to 5 , player 2 and player 3 will be more willing to be in league with player 1 . As a result, the probability of Lianheng 1 and Lianheng 2 increase. Also, it changes from $r_{2}+r_{3}>r_{1}$ to $r_{2}+r_{3}=r_{1}$, so the probability of Hezong decreases.

(2) From $(4,3,2)$ to $(4,3,2.5)$ :

If $r_{1}$ and $r_{2}$ remain constant, $r_{3}$ rises to 2.5 . Because $r_{3}$ become larger, player 1 and player 2 will be more willing to be in league with player 3, and the probability of Lianheng 2 and Hezong will increase. In the meanwhile, the probability of Lianheng 1 will decrease.

(3) From $(4,3,2)$ to $(5,3,2.5)$ :

Now $r_{2}$ is still constant, but $r_{1}$ and $r_{3}$ rise. For strength of player 1 and 3 becomes larger, they will have more reason to league together. Therefore, the probability of Lianheng 2 increases, but the probability of Lianheng 1 and Hezong decreases.

(4) From $(5,3,2)$ to $(4,3,2.5)$ :

While $r_{1}$ falls, $r_{2}$ remains constant and $r_{3}$ raises, the likelihood of player 1 and 2 league together decreases, but player 2 and 3 increases. Also, it changes from $r_{2}+r_{3}=r_{1}$ to $r_{2}+r_{3}>r_{1}$, so player 2 and 3 will be more willing to league together. Thus, the probability of Lianheng 1 decreases but the probability of Hezong increases. And the value of $r_{1}+r_{3}$ falls from 7 to 6.5 , so the probability of Lianheng 2 decreases.

(5) From $(5,3,2)$ to $(5,3,2.5)$ :

Here $r_{1}$ and $r_{2}$ remain constant, and $r_{3}$ rises. It changes from $r_{2}+r_{3}=r_{1}$ to $r_{2}+r_{3}>r_{1}$, so player 2 and 3 will be more willing to be league together. Therefore, the probability of Hezong increases. In the meanwhile, the probability of Lianheng 1 and Lianheng 2 decreases.

(6) From $(4,3,2.5)$ to $(5,3,2.5)$ :

Now $r_{1}$ rises, $r_{2}$ and $r_{3}$ are constant. Because $r_{1}$ increases, player 2 and 3 will be more willing to be in league with player 1 . As a result, the probability of Lianheng 1 and Lianheng 2 increase. And $r_{1}$ is getting closer to $r_{2}+r_{3}$, so the probability of Hezong decreases.

In a word, the probability of Lianheng 1 and Lianheng 2 would increase when $r_{1}$ increases. The probability of 
Lianheng 2 and Hezong would increase while $r_{3}$ increases. If both $r_{1}$ and $r_{3}$ increase, the probability of

Lianheng 2 would increase. But if $r_{1}$ decreases and $r_{3}$ increases, the probability of Lianheng 1 would decrease and the probability of Hezong would increase. That is, the probability of coalition formation would be higher while the player is stronger.

In Chinese history, the fall of Jin happened in 1234. The location of Mongol was in the north of Jin, and Southern Song was in south to Jin. Among the three kingdoms, strength of Mongol was the strongest, Jin was the next, and Southern Song was the weakest. Maybe because of the location, Mongol was in league with Southern Song, so as to attack Jin from both sides. And the result was Mongol and Southern Song frustrated Jin. This corresponds to Lianheng 2 in our paper.

As it is shown in Fig. 5, the probability of Lianheng 2 when $b=1$ is larger than that when $b=2$. And the probability of Lianheng 2 when $b=2$ is also larger than that when $b=3$ in each strength vector. When $r_{2}$,

$r_{3}$ and $b$ remain constant, the probability of Lianheng 2 will be higher if $r_{1}$ is larger. But when $r_{1}, r_{2}$ and $b$ are constant, the probability of Lianheng 2 will be higher if $r_{3}$ is smaller. In other words, the probability of

Lianheng 2 will be higher if $b$ falls, $r_{1}$ rises or $r_{3}$ falls. It means that the probability of Lianheng 2 will be higher when the cost of the war is lower and the strength of player 1 and player 3 is farther.

Another famous example in Chinese history was the Battle of Red Cliffs in 208. It's a battle of three kingdoms, Cao Cao, Sun Quan and Liu Bei. (Note 9) The order of the strength from the strongest to the weakest is Cao Cao, Sun Quan and Liu Bei. However, Sun Quan and Liu Bei chose to league together in this battle and defeated Cao Cao. And it corresponds to Hezong in our paper.

As it is shown in Fig. 6, the probability of Hezong when $b=3$ is larger than that when $b=2$. And the probability of Hezong when $b=2$ is larger than that when $b=1$ in each strength vector. When $r_{2}, r_{3}$

and $b$ are constant, the probability of Hezong will be higher if $r_{1}$ is smaller. But while $r_{1}, r_{2}$ and $b$ are constant, the probability of Hezong will be higher if $r_{3}$ is larger. Namely, the probability of Hezong will be higher if $b$ rises, $r_{1}$ falls or $r_{3}$ raises. It means that the probability of Hezong will be higher when the cost of the war is higher and the strength of player 1 and player 3 is closer.

According to the introduction, player 1 and player 2 league together or player 1 and player 3 league together is Lianheng, and it corresponds to Lianheng 1 and Lianheng 2 in our paper. Besides, player 2 and player 3 league together is called Hezong.

Corollary. In our paper, the fall of Jin corresponds to Lianheng 2, and the Battle of Red Cliffs corresponds to Hezong. But Lianheng 2 and Hezong exist in mixed strategy Nash equilibriums merely. Therefore, it is possible that the Battle of Red Cliffs and the fall of Jin are just coincident or random events.

\section{Sequential move game}

Now we discuss condition that players choose their strategies sequentially. It's a game of perfect information, and players know exactly where they are. We take player 2 moves first for example, as it is shown in Fig. 7 . For 
player 1 , he has two chances to select. If he selects $\ell_{2}$ at node $y$ and chooses $\ell_{3}$ at node $w$, then his strategy will be $\ell_{2} \ell_{3}$. If player 2 selects $\ell_{1}$ at node $x$, his strategy will be $\ell_{1}$. And if player 3 selects $\ell_{1}$ at node $v$ and chooses $\ell_{2}$ at node $z$, then his strategy will be $\ell_{1} \ell_{2}$. About the case of player 2 starts first, there are four SPE as follows:

\section{Insert Figure 7 Here}

(1) $\left(\ell_{2} \ell_{2}, \ell_{1}, \ell_{2} \ell_{1}\right)$

At node $z$, player 3 will choose $\ell_{1}$ if $a_{23} \geq a_{43}$, which can be simplified to $r_{3} \leq[a /(a+2 \delta b)] r_{1}$. And player 1 chooses $\ell_{2}$ at node $y$ because $a_{11} \geq a_{21}$. At node $w$, player 1 chooses $\ell_{2}$ because $a_{41} \geq a_{21}$. And player 3 will choose $\ell_{2}$ at node $v$ if $a_{33} \geq a_{43}$, which can be simplified to $r_{3} \leq[a /(a+2 \delta b)] r_{2}$. Then, player 2 chooses $\ell_{1}$ at node $x$ because $a_{12} \geq a_{32}$. As a result, the subgame perfect Nash equilibrium is Lianheng 1.

(2) $\left(\ell_{2} \ell_{2}, \ell_{1}, \ell_{1} \ell_{1}\right)$

At node $z$, player 3 will choose $\ell_{1}$ if $a_{23} \geq a_{43}$, which can be simplified to $r_{3} \leq[a /(a+2 \delta b)] r_{1}$. And player 1 chooses $\ell_{2}$ at node $y$ because $a_{11} \geq a_{21}$. At node $w$, player 1 chooses $\ell_{2}$ because $a_{41} \geq a_{21}$. And player 3 will choose $\ell_{1}$ at node $v$ if $a_{43} \geq a_{33}$, which can be simplified to $r_{3} \geq[a /(a+2 \delta b)] r_{2}$. Then, player 2 will choose $\ell_{1}$ at node $x$ if $a_{12} \geq a_{42}$, which can be simplified to $r_{2} \leq[a /(a+2 \delta b)] r_{1}$. Therefore, the subgame perfect Nash equilibrium is Lianheng 1.

(3) $\left(\ell_{2} \ell_{2}, \ell_{3}, \ell_{1} \ell_{1}\right)$

At node $z$, player 3 will choose $\ell_{1}$ if $a_{23} \geq a_{43}$, which can be simplified to $r_{3} \leq[a /(a+2 \delta b)] r_{1}$. And player 1 chooses $\ell_{2}$ at node $y$ because $a_{11} \geq a_{21}$. At node $w$, player 1 chooses $\ell_{2}$ because $a_{41} \geq a_{21}$. And player 3 will choose $\ell_{1}$ at node $v$ if $a_{43} \geq a_{33}$, which can be simplified to $r_{3} \geq[a /(a+2 \delta b)] r_{2}$. Then, player 2 will choose $\ell_{3}$ at node $x$ if $a_{42} \geq a_{12}$, which can be simplified to 
$r_{2} \geq[a /(a+2 \delta b)] r_{1}$. Consequently, the subgame perfect Nash equilibrium is Singleton.

(4) $\left(\ell_{3} \ell_{2}, \ell_{1}, \ell_{1} \ell_{2}\right)$ or $\left(\ell_{3} \ell_{2}, \ell_{3}, \ell_{1} \ell_{2}\right)$

At node $z$, player 3 will choose $\ell_{2}$ if $a_{43} \geq a_{23}$, which can be simplified to $r_{3} \geq[a /(a+2 \delta b)] r_{1}$. And player 1 chooses $\ell_{3}$ at node $y$ because $a_{41} \geq a_{11}$. At node $w$, player 1 chooses $\ell_{2}$ because $a_{41} \geq a_{21}$. And player 3 will choose $\ell_{1}$ at node $v$ if $a_{43} \geq a_{33}$, which can be simplified to $r_{3} \geq[a /(a+2 \delta b)] r_{2}$. Then, player 2 will choose $\ell_{1}$ or $\ell_{3}$ at node $x$ because $a_{42}=a_{42}$. Hence, the subgame perfect Nash equilibrium is Singleton.

The analyses of player 1 moves first and player 3 moves first are the same. And the outcome is shown in Table 6 . From the table, we discover that no matter who moves first, Lianheng 1 and Singleton exist. That is, player 1 will be in league with player 2 or singleton league exists in sequential move game.

Equilibriums and states of Table 6 which correspond to the regions of Fig. 2 are shown in Table 7. From Fig. 3 and Table 7, Lianheng 1 and Singleton both exists in region $A_{9}$. If we rise either $r_{2}$ or $r_{3}$, the outcome is still Lianheng 1 and Singleton. But if we increase both $r_{2}$ and $r_{3}$ at the same time, it will exist Singleton merely as in region $A_{3}, A_{4}$ and $A_{5}$. In other words, if strength of three players is getting stronger and closer, singleton league will happen.

Proposition 2. When three players move sequentially,

(1) for player 1, in order to increase his winning rate, he has a tendency to be in league with player 2.

(2) for player 2 and 3 , if $r_{2}>[a /(a+2 \delta b)] r_{1}$ and $r_{3}>[a /(a+2 \delta b)] r_{2}$, it will only exist Singleton. Otherwise, both Lianheng 1 and Singleton exist.

In this game, we know that pure strategy Nash Equilibriums of sequential move and pure strategy Nash Equilibriums of simultaneous move are the same. It exists only Lianheng 1 and Singleton. Nevertheless, Lianheng 1, Lianheng 2, Hezong and Singleton all exist in mixed strategy Nash Equilibriums when three players move simultaneously.

\section{Conclusion}

In this paper, we know that Lianheng 2 and Hezong exist in mixed strategy Nash equilibriums of simultaneous move game merely. Since the outcome of mixed strategy is random, we couldn't predict it in advance. Thus, it is likely that the fall of Jin and the Battle of Red Cliffs in Chinese history were coincidences. On the other hand, Lianheng 1 and Singleton exists in both simultaneous and sequential move game. Because the equilibriums of Lianheng are more than that of Hezong, we can conclude that players tend to be in league with the strongest mostly.

But Tan and Wang (1997) assume there is only one final winner who gets the prize and all the other players receive nothing. Hence, weaker players have a tendency to league together to defeat the strongest first, and then they further fight among themselves. In our paper, we suppose the survivor can get a fixed amount of profit in each period. The result is that Lianheng 1 exists in pure strategy Nash equilibriums of simultaneous move game 
when the strength of player 2 is weaker.

However, there are still problems to be solved or improved. Is there a better setting for the winning rate? Contrary to peace, it takes more time in a war. For that reason, making a difference of time between peace and a war may be needed. There are only three players in our paper, but actually the seven warring states in Chinese history seems to be a seven-player game. Consequently, we can extend the case to four or more players. And if we can get real strength of countries, it is also interesting to substitute that to our model.

\section{Appendix A}

When player 1 and 2 league together, player 1's winning rate is $\left(r_{1}+r_{2}\right) /\left(r_{1}+r_{2}+r_{3}\right)$ in the first period, and $r_{1} /\left(r_{1}+r_{2}\right)$ in the second period. Also, he gets $a / 2$ in the first period, and $v_{1}$ in the second period.

Player 2 is similar to player 1. But player 3's winning rate is $r_{3} /\left(r_{1}+r_{2}+r_{3}\right)$, and he gets $a$ in the first period and gets $v_{2}$ in the second period. $\delta$ is the discount factor. And the payoffs of the following leagues come out by the same method.

$$
\begin{aligned}
& a_{11}: \frac{r_{1}+r_{2}}{r_{1}+r_{2}+r_{3}}\left[\frac{a}{2}+\frac{r_{1}}{r_{1}+r_{2}} \delta(a+v)\right]=\frac{r_{1}+r_{2}}{r_{1}+r_{2}+r_{3}} \frac{a}{2}+\frac{r_{1}}{r_{1}+r_{2}+r_{3}} \frac{\delta(a+\delta b)}{1-\delta} \\
& a_{12}: \frac{r_{1}+r_{2}}{r_{1}+r_{2}+r_{3}}\left[\frac{a}{2}+\frac{r_{2}}{r_{1}+r_{2}} \delta(a+v)\right]=\frac{r_{1}+r_{2}}{r_{1}+r_{2}+r_{3}} \frac{a}{2}+\frac{r_{2}}{r_{1}+r_{2}+r_{3}} \frac{\delta(a+\delta b)}{1-\delta} \\
& a_{13}: \frac{r_{3}}{r_{1}+r_{2}+r_{3}}(a+\delta v)=\frac{r_{3}}{r_{1}+r_{2}+r_{3}} \frac{a+\delta b}{1-\delta}
\end{aligned}
$$

When player 1 and 3 league together,

$$
\begin{aligned}
& a_{21}: \frac{r_{1}+r_{3}}{r_{1}+r_{2}+r_{3}}\left[\frac{a}{2}+\frac{r_{1}}{r_{1}+r_{3}} \delta(a+v)\right]=\frac{r_{1}+r_{3}}{r_{1}+r_{2}+r_{3}} \frac{a}{2}+\frac{r_{1}}{r_{1}+r_{2}+r_{3}} \frac{\delta(a+\delta b)}{1-\delta} \\
& a_{22}: \frac{r_{2}}{r_{1}+r_{2}+r_{3}}(a+\delta v)=\frac{r_{2}}{r_{1}+r_{2}+r_{3}} \frac{a+\delta b}{1-\delta} \\
& a_{23}: \frac{r_{1}+r_{3}}{r_{1}+r_{2}+r_{3}}\left[\frac{a}{2}+\frac{r_{1}}{r_{1}+r_{3}} \delta(a+v)\right]=\frac{r_{1}+r_{3}}{r_{1}+r_{2}+r_{3}} \frac{a}{2}+\frac{r_{3}}{r_{1}+r_{2}+r_{3}} \frac{\delta(a+\delta b)}{1-\delta}
\end{aligned}
$$

When player 2 and 3 league together,

$$
\begin{aligned}
& a_{31}: \frac{r_{1}}{r_{1}+r_{2}+r_{3}}(a+\delta v)=\frac{r_{1}}{r_{1}+r_{2}+r_{3}} \frac{a+\delta b}{1-\delta} \\
& a_{32}: \frac{r_{2}+r_{3}}{r_{1}+r_{2}+r_{3}}\left[\frac{a}{2}+\frac{r_{1}}{r_{1}+r_{3}} \delta(a+v)\right]=\frac{r_{2}+r_{3}}{r_{1}+r_{2}+r_{3}} \frac{a}{2}+\frac{r_{2}}{r_{1}+r_{2}+r_{3}} \frac{\delta(a+\delta b)}{1-\delta}
\end{aligned}
$$




$$
a_{33}: \frac{r_{2}+r_{3}}{r_{1}+r_{2}+r_{3}}\left[\frac{a}{2}+\frac{r_{1}}{r_{1}+r_{3}} \delta(a+v)\right]=\frac{r_{2}+r_{3}}{r_{1}+r_{2}+r_{3}} \frac{a}{2}+\frac{r_{3}}{r_{1}+r_{2}+r_{3}} \frac{\delta(a+\delta b)}{1-\delta}
$$

When there is singleton league,

$$
\begin{aligned}
& a_{41}: \frac{r_{1}}{r_{1}+r_{2}+r_{3}}(a+\delta v)=\frac{r_{1}}{r_{1}+r_{2}+r_{3}} \frac{a+\delta b}{1-\delta} \\
& a_{42}: \frac{r_{2}}{r_{1}+r_{2}+r_{3}}(a+\delta v)=\frac{r_{2}}{r_{1}+r_{2}+r_{3}} \frac{a+\delta b}{1-\delta} \\
& a_{43}: \frac{r_{3}}{r_{1}+r_{2}+r_{3}}(a+\delta v)=\frac{r_{3}}{r_{1}+r_{2}+r_{3}} \frac{a+\delta b}{1-\delta}
\end{aligned}
$$

\section{Appendix B}

Take Lianheng 1 for example. If player 1 and 2 league together is Nash equilibrium, it will correspond to condition (1) and (5) in Fig. 2. We analyze condition (1) initially. The strategy profile is $\left(\ell_{2}, \ell_{1}, \ell_{1}\right)$, and the payoff is $\left(a_{11}, a_{12}, a_{13}\right)$. For player 1 , choosing $\ell_{2}$ is better than $\ell_{3}$. It represents that $a_{11} \geq a_{21}$, which can be simplified to $r_{2} \geq r_{3}$, and it is definitely true. For player 2, choosing $\ell_{1}$ is better than $\ell_{3}$ if $a_{12} \geq a_{42}$, which can be simplified to $r_{2} \leq[a /(a+2 \delta b)] r_{1}$, and it's uncertain. For player 3 , choosing $\ell_{1}$ is better than $\ell_{2}$ because $a_{13} \geq a_{13}$ is definitely true.

Then, we discuss condition (5). The strategy profile is $\left(\ell_{2}, \ell_{1}, \ell_{2}\right)$, and the payoff is $\left(a_{11}, a_{12}, a_{13}\right)$. For player 1 , choosing $\ell_{2}$ is better than $\ell_{3}$. But $a_{11} \geq a_{41}$, which can be simplified to $r_{1} \leq[a /(a+2 \delta b)] r_{2}$, is a contradiction. For player 2 , choosing $\ell_{1}$ is better than $\ell_{3}$. And $a_{12} \geq a_{32}$, which can be simplified to $r_{1} \geq r_{3}$, is definitely true. For player 3, choosing $\ell_{2}$ is better than $\ell_{1}$. Also, $a_{13} \geq a_{13}$ is definitely true.

And methods of analyzing Lianheng 2, Hezong, and Singleton are the same. Then, we can conclude that it is likely that condition (1), (3) and (6) are Nash equilibriums, but condition (2), (4), (5), (7) and (8) are not.

\section{Appendix C}

While three players all take mixed strategy, it is indifferent for players to select between their choices. For player 1 , choosing $\ell_{2}$ is as good as $\ell_{3}$, and things like that. Then we will have the following equations:

$$
a_{41}(t-u)=a_{11} t-a_{21} u(\text { Note } 10)
$$




$$
\begin{aligned}
& a_{42}(u+s-1)=a_{12} s-a_{32}(1-u)(\text { Note } 11) \\
& a_{43}(t-s)=a_{23}(1-s)-a_{33}(1-t)(\text { Note } 12)
\end{aligned}
$$

Equations (C1), (C2) and (C3) imply

$$
\begin{aligned}
& s=\frac{\left[2 b \delta r_{2}+a\left(r_{2}-r_{3}\right)\right]\left\{2 a b \delta\left(r_{1}-r_{3}\right)^{2}-4 b^{2} \delta^{2} r_{1} r_{3}+a^{2}\left[r_{1}^{2}+r_{2}^{2}-r_{2} r_{3}+r_{3}^{2}-r_{1}\left(r_{2}+r_{3}\right)\right]\right\}}{2 b \delta\left\{\left(a^{2}+2 a b \delta\right)\left[\left(r_{1}^{2}+r_{2} r_{3}\right)\left(r_{2}+r_{3}\right)+r_{1}\left(r_{2}^{2}-6 r_{2} r_{3}+r_{3}^{2}\right)-8 b^{2} \delta^{2} r_{1} r_{2} r_{3}\right]\right\}} \\
& t=\frac{\left[2 b \delta r_{1}+a\left(r_{1}-r_{3}\right)\right]\left\{2 a b \delta\left(r_{2}-r_{3}\right)^{2}-4 b^{2} \delta^{2} r_{2} r_{3}+a^{2}\left[r_{1}^{2}+r_{2}^{2}-r_{2} r_{3}+r_{3}^{2}-r_{1}\left(r_{2}+r_{3}\right)\right]\right\}}{2 b \delta\left\{a(a+2 b \delta) r_{1} r_{2}\left(r_{1}+r_{2}\right)+\left[a(a+2 b \delta)\left(r_{1}^{2}-3 r_{1} r_{2}+r_{2}^{2}\right)-8 b^{2} \delta^{2} r_{1} r_{2}\right] r_{3}+a(a+2 b \delta)\left(r_{1}+r_{2}\right) r_{3}^{2}\right\}} \\
& u=\frac{\left[2 b \delta r_{1}+a\left(r_{1}-r_{2}\right)\right]\left\{2 a b \delta\left(r_{2}-r_{3}\right)^{2}-4 b^{2} \delta^{2} r_{2} r_{3}+a^{2}\left[r_{1}^{2}+r_{2}^{2}-r_{2} r_{3}+r_{3}^{2}-r_{1}\left(r_{2}+r_{3}\right)\right]\right\}}{2 b \delta\left\{\left(a^{2}+2 a b \delta\right)\left[\left(r_{1}^{2}+r_{2} r_{3}\right)\left(r_{2}+r_{3}\right)+r_{1}\left(r_{2}^{2}-6 r_{2} r_{3}+r_{3}^{2}\right)\right]-8 b^{2} \delta^{2} r_{1} r_{2} r_{3}\right\}}
\end{aligned}
$$

For example, when $\delta=0.8, a=1, b=1$ and $\left(r_{1}, r_{2}, r_{3}\right)=(4,3,2)$, it will result in that $s=0.5279$, $t=0.7425, u=0.6541$.

\section{References}

Beest, I. V., Dijk, E. V., \& Wilke, H. (2004a). The Interplay of Self-Interest and Equity in Coalition Formation. European Journal of Social Psychology, 34(5), 547-565.

Beest, I. V., Dijk, E. V., \& Wilke, H. (2004b). Resources and Alternatives in Coalition Formation: The Effects on Payoff, Self-Serving Behavior, and Bargaining Length. European Journal of Social Psychology, 34(6), 713-728.

Beest, I. V., Dijk, E. V., Dreu, C. K. W. D., \& Wilke, H. (2005). Do-No-Harm in Coalition Formation: Why Losses Inhibit Exclusion and Promote Fairness Cognitions. Journal of Experimental Social Psychology, 41(6), 609-617.

Beest, I. V., Wilke, H., \& Dijk, E. V. (2003). The Excluded Player in Coalition Formation. Personality and Social Psychology Bulletin, 29(2), 237-247.

Bottom, W. P., Holloway, McClurg, J., \& Miller, G. J. (2000). Negotiating a Coalition: Risk, Quota Shaving, and Learning to Bargain. Journal of Conflict Resolution, 44(2), 147-169.

Eguia, J. X. (2006). Voting Blocs, Coalition and Parties. Social Science Working Paper 1257.

Eguia, J. X. (2007). United We Vote. Journal of Public Economic Theory, 9(4), 607-639.

Esteban, J., Sákovics, J. (2003). Olson vs. Coase: Coalitional Worth in Conflict. Theory and Decision, 55(4), 339-357.

Feix, M. R., Lepelley, D., Merlin, V. R., \& Rouet, J.-L. (2007). On the Voting Power of an Alliance and the Subsequent Power of Its Members. Social Choice and Welfare, 28(2), 181-207.

Ikonnikova, S. (2007). Coalition Formation, Bargaining and Investments in Networks with Externalities: Analysis of the Eurasian Gas Supply Network. Munich Personal RePEc Archive (MPRA) Paper 915.

Konrad, K. A., \& Kovenock, D. (2008). The Alliance Formation Puzzle and Capacity Constraints. Discussion Paper SP II 2008 - 07, Wissenschaftszentrum Berlin.

Neary, H. M. (1996). To Fight or Not to Fight: Equilibrium Structure in a Conflict Model when Conflict is Costly. Mimeo, Department of Economics, University of British Columbia.

Ridder, A. D., \& Rusinowska, A. (2008). On Some Procedures of Forming a Multipartner Alliance. Journal of Economics \& Management Strategy, 17(2), 443-487.

Skaperdas, S. (1996). Contest Success Functions. Economic Theory, 7(2), 283-290. 
Skaperdas, S. (1998). On the Formation of Alliances in Conflict and Contests. Public Choice, 96(1-2), 25-42.

Sorokin, G. L. (1994). Alliance Formation and General Deterrence: A Game-Theoretic Model and the Case of Israel. Journal of Conflict Resolution, 38(2), 298-325.

Tan, G., \& Wang, R. (1997). Endogenous coalition formation in rivalry. Queen's Economics Department Working Paper 956.

\section{Notes}

Note 1. For example, if player 1 chooses to be in league with player 2 , player 2 chooses to be in league with player 3, and player 3 chooses to be in league with player 1, then it will result in singleton league.

Note 2. The form of winning rate is commonly used in the literature by Skaperdas (1996).

Note 3. The payoff values are shown in Appendix A.

Note 4. The details of pure strategy equilibriums are shown in Appendix B.

Note 5. $s, t, u$ of Equilibrium 4 are shown in Appendix C.

Note 6. $u=\left[2 b d r_{1}+a\left(r_{1}-r_{2}\right)\right] /\left[2 b d r_{1}+a\left(r_{1}-r_{3}\right)\right], \quad s \leq\left\{\left[2 b d r_{2}+a\left(r_{2}-r_{3}\right)\right](1-u)\right\} /\left[2 b d r_{2}-a\left(r_{1}-r_{2}\right)\right]$.

Note 7. $u=a\left(r_{1}-r_{3}\right) /\left[2 b d r_{2}+a\left(r_{2}-r_{3}\right)\right], t \leq\left\{\left[2 b d r_{1}+a\left(r_{1}-r_{3}\right)\right] u\right\} /\left[2 b d r_{1}+a\left(r_{1}-r_{2}\right)\right]$.

Note 8. $t \leq\left[2 b d r_{1}+a\left(r_{1}-r_{3}\right)\right] /\left[2 b d r_{1}+a\left(r_{1}-r_{2}\right)\right]$.

Note 9. Cao Cao, Sun Quan and Liu Bei form countries later as Cao Wei, Eastern Wu and Shu Han respectively.

Note 10. It simplifies from $a_{11} t u+a_{41}(1-t) u+a_{11} t(1-u)+a_{31}(1-t)(1-u)=a_{21} t u+a_{21}(1-t) u+a_{41} t(1-u)+a_{31}(1-t)(1-u)$.

Note 11. It simplifies from $a_{12} s u+a_{22}(1-s) u+a_{12} s(1-u)+a_{42}(1-s)(1-u)=a_{42} s u+a_{22}(1-s) u+a_{32} s(1-u)+a_{32}(1-s)(1-u)$, and it stands when $r_{2} \geq[a /(a+2 \delta b)] r_{1}$.

Note 12. It simplifies from $a_{13} s t+a_{23}(1-s) t+a_{43} s(1-t)+a_{23}(1-s)(1-t)=a_{13} s t+a_{43}(1-s) t+a_{33} s(1-t)+a_{33}(1-s)(1-t)$, and it stands when $r_{3} \geq[a /(a+2 \delta b)] r_{1}$ or $r_{3} \leq[a /(a+2 \delta b)] r_{2}$.

Table 1. Nash equilibriums

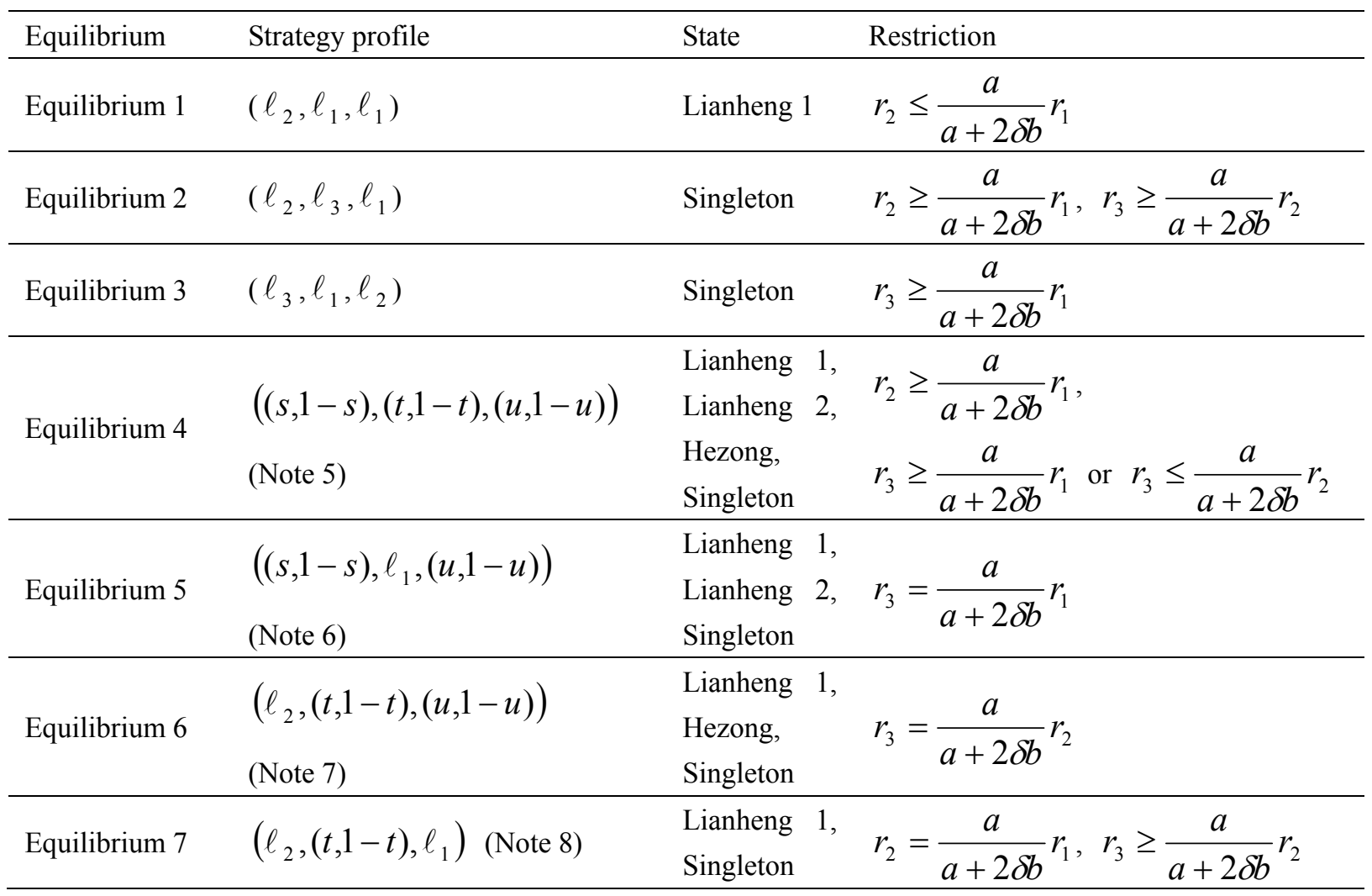


Table 2. Scope of the eleven regions

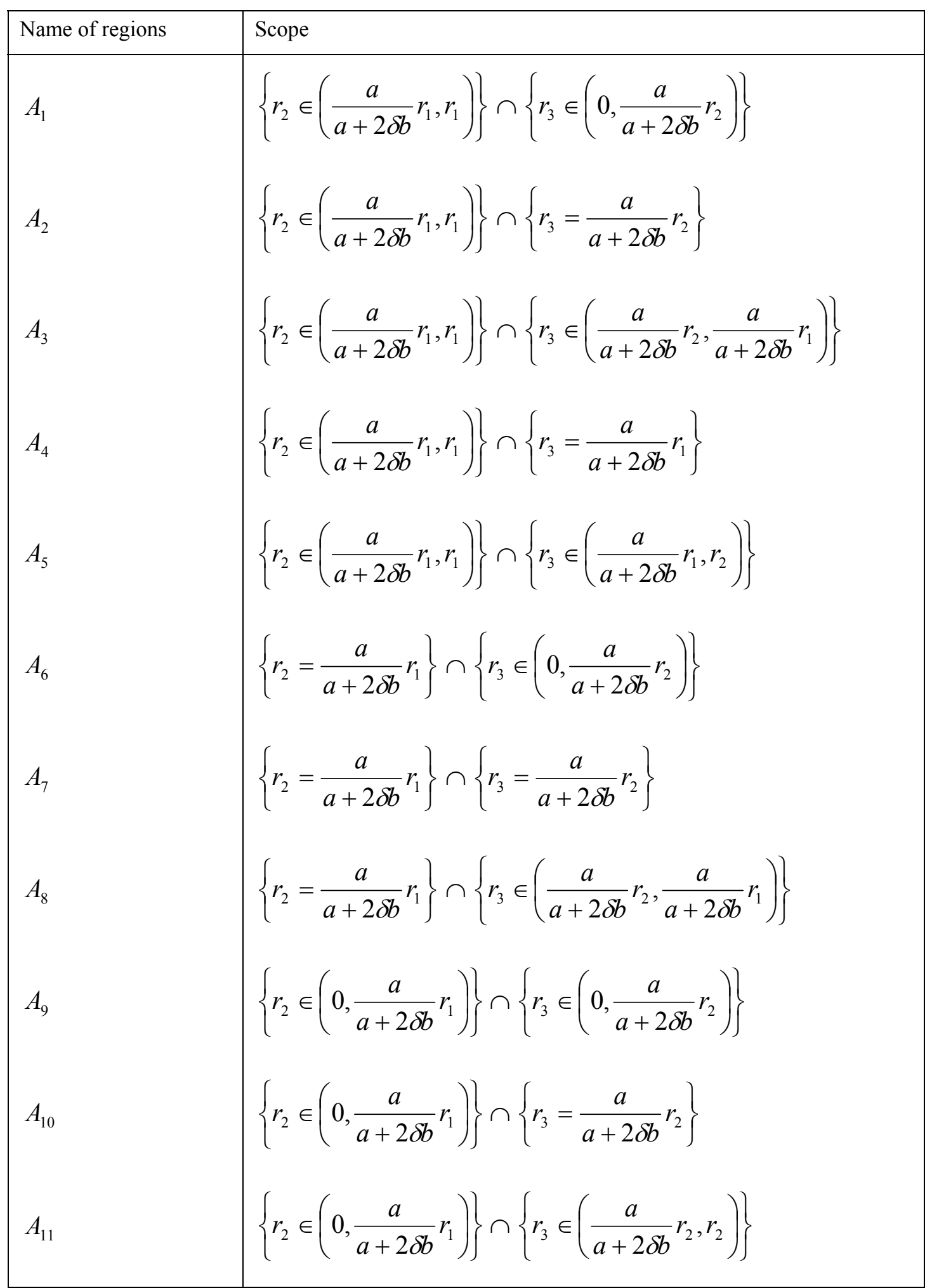


Table 3. Nash equilibriums and states of the eleven regions

\begin{tabular}{|c|c|c|}
\hline Name of regions & Nash equilibrium & State \\
\hline$A_{1}$ & Equilibrium 4 & Lianheng 1, Lianheng 2, Hezong, Singleton \\
\hline$A_{2}$ & Equilibrium 2, 4, 6 & Lianheng 1, Lianheng 2, Hezong, Singleton \\
\hline$A_{3}$ & Equilibrium 2 & Singleton \\
\hline$A_{4}$ & Equilibrium 2, 3, 4, 5 & Lianheng 1, Lianheng 2, Hezong, Singleton \\
\hline$A_{5}$ & Equilibrium 2, 3, 4 & Lianheng 1, Lianheng 2, Hezong, Singleton \\
\hline$A_{6}$ & Equilibrium 1, 4 & Lianheng 1, Lianheng 2, Hezong, Singleton \\
\hline$A_{7}$ & Equilibrium 1, 2, 4, 6, 7 & Lianheng 1, Lianheng 2, Hezong, Singleton \\
\hline$A_{8}$ & Equilibrium 1, 2, 7 & Lianheng 1, Singleton \\
\hline$A_{9}$ & Equilibrium 1 & Lianheng 1 \\
\hline$A_{10}$ & Equilibrium 1, 6 & Lianheng 1, Hezong, Singleton \\
\hline$A_{11}$ & Equilibrium 1 & Lianheng 1 \\
\hline
\end{tabular}

Table 4 . The value of $s, t, u$

\begin{tabular}{|c|c|c|c|c|c|}
\hline & $\left(r_{1}, r_{2}, r_{3}\right)$ & $(4,3,2)$ & $(5,3,2)$ & $(4,3,2.5)$ & $(5,3,2.5)$ \\
\hline \multirow{3}{*}{$b=1$} & $S$ & 0.5317 & 0.3970 & 0.5262 & 0.4948 \\
\hline & $t$ & 0.7118 & 0.8608 & 0.6446 & 0.7474 \\
\hline & $u$ & 0.6345 & 0.7891 & 0.6076 & 0.7149 \\
\hline \multirow{3}{*}{$b=2$} & $S$ & 0.5244 & 0.4981 & 0.5146 & 0.5053 \\
\hline & $t$ & 0.6011 & 0.6600 & 0.5717 & 0.6217 \\
\hline & $u$ & 0.5645 & 0.6286 & 0.5537 & 0.6065 \\
\hline \multirow{3}{*}{$b=3$} & $S$ & 0.5175 & 0.5033 & 0.5100 & 0.5046 \\
\hline & $t$ & 0.5667 & 0.6047 & 0.5477 & 0.5808 \\
\hline & $u$ & 0.5427 & 0.5845 & 0.5358 & 0.5710 \\
\hline
\end{tabular}


Table 5. The probability of four states

\begin{tabular}{|c|c|c|c|c|c|}
\cline { 2 - 6 } \multicolumn{1}{c|}{} & $\left(r_{1}, r_{2}, r_{3}\right)$ & $(4,3,2)$ & $(5,3,2)$ & $(4,3,2.5)$ & $(5,3,2.5)$ \\
\hline \multirow{5}{*}{$b=1$} & Lianheng 1 & 0.3785 & 0.3417 & 0.3392 & 0.3698 \\
\cline { 2 - 6 } & Lianheng 2 & 0.2971 & 0.4758 & 0.2879 & 0.3612 \\
\cline { 2 - 6 } & Hezong & 0.1053 & 0.0293 & 0.1394 & 0.0720 \\
\cline { 2 - 6 } & Singleton & 0.2191 & 0.1531 & 0.2335 & 0.1970 \\
\hline \multirow{5}{*}{$b=2$} & Lianheng 1 & 0.3152 & 0.3287 & 0.2942 & 0.3141 \\
\cline { 2 - 6 } & Lianheng 2 & 0.2685 & 0.3155 & 0.2688 & 0.3001 \\
\cline { 2 - 6 } & Hezong & 0.1737 & 0.1263 & 0.1911 & 0.1489 \\
\cline { 2 - 6 } & Singleton & 0.2426 & 0.2295 & 0.2459 & 0.2370 \\
\hline & Lianheng 1 & 0.2933 & 0.3043 & 0.2793 & 0.2931 \\
\cline { 2 - 6 } & Lianheng 2 & 0.2619 & 0.2903 & 0.2625 & 0.2829 \\
\cline { 2 - 6 } & Hezong & 0.1981 & 0.1642 & 0.2100 & 0.1798 \\
\cline { 2 - 6 } & Singleton & 0.2467 & 0.2411 & 0.2482 & 0.2442 \\
\hline
\end{tabular}

Table 6. Subgame Perfect Nash equilibriums

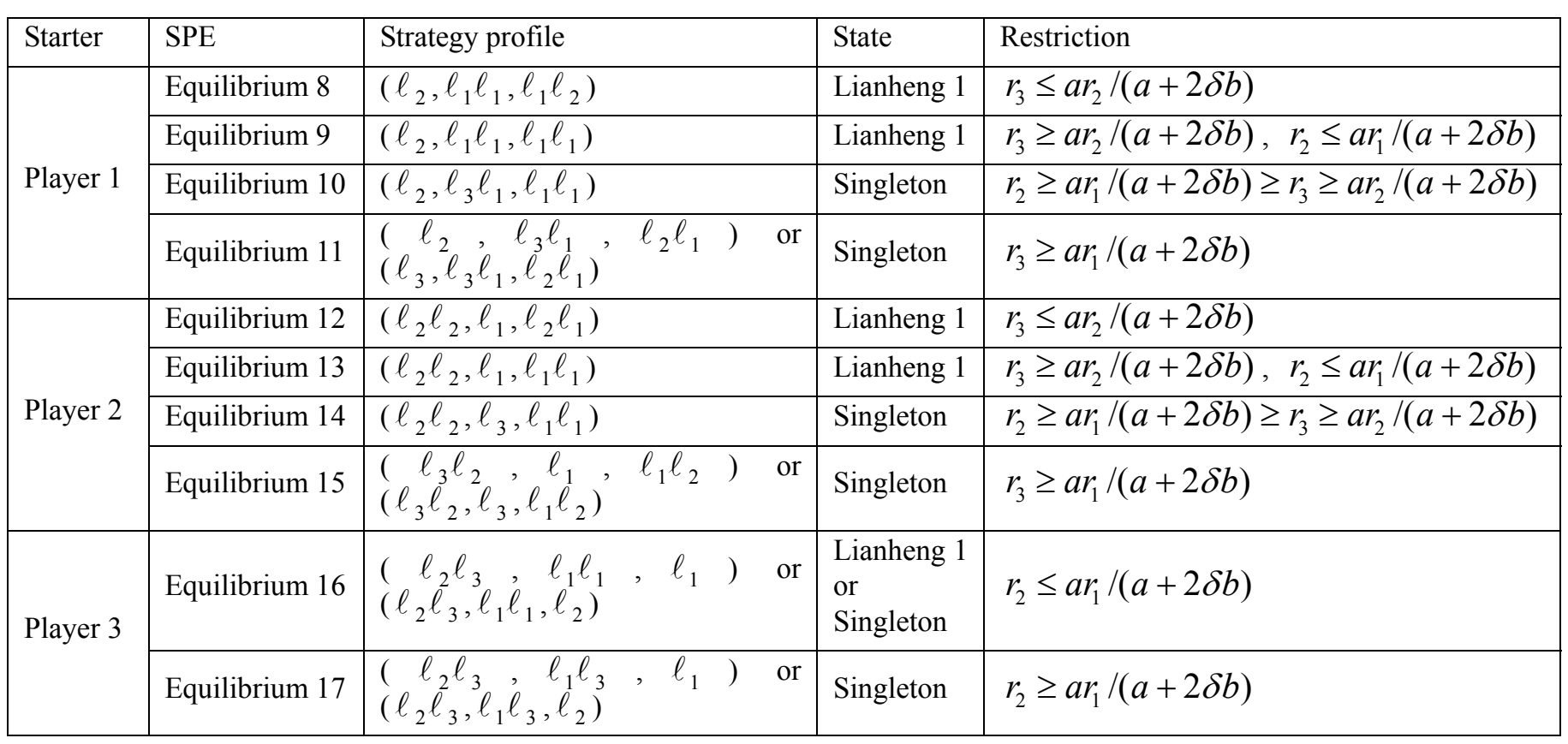


Table 7. SPE and states of the eleven regions

\begin{tabular}{cll}
\hline Name of regions & SPE & State \\
\hline$A_{1}$ & Equilibrium 8, 12,17 & Lianheng 1, Singleton \\
$A_{2}$ & Equilibrium 8, 10,12,14,17 & Lianheng 1, Singleton \\
$A_{3}$ & Equilibrium 10,14,17 & Singleton \\
$A_{4}$ & Equilibrium 10, 11, 14, 15,17 & Singleton \\
$A_{5}$ & Equilibrium 11, 15, 17 & Singleton \\
$A_{6}$ & Equilibrium 8, 12,16,17 & Lianheng 1, Singleton \\
$A_{7}$ & Equilibrium 8, 9, 10,12,13,14, 16,17 & Lianheng 1, Singleton \\
$A_{8}$ & Equilibrium 9, 10, 13,14, 16,17 & Lianheng 1, Singleton \\
$A_{9}$ & Equilibrium 8, 12,16 & Lianheng 1, Singleton \\
$A_{10}$ & Equilibrium 8, 9, 12,13,16 & Lianheng 1, Singleton \\
$A_{11}$ & Equilibrium 9, 13,16 & Lianheng 1, Singleton \\
\hline
\end{tabular}

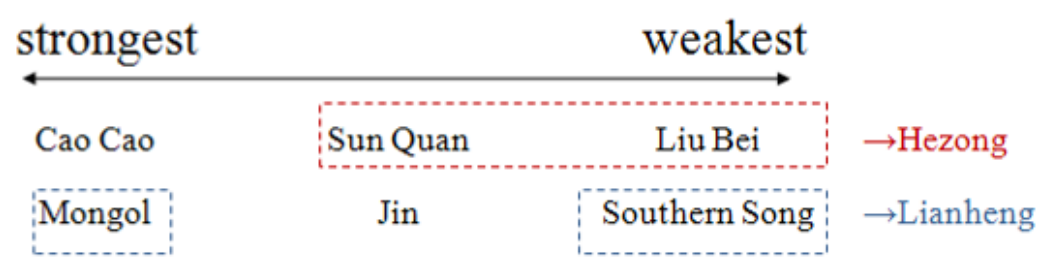

Figure 1. Examples of Hezong and Lianheng

$$
\begin{aligned}
& \text { player } 2 \quad \text { player } 2
\end{aligned}
$$

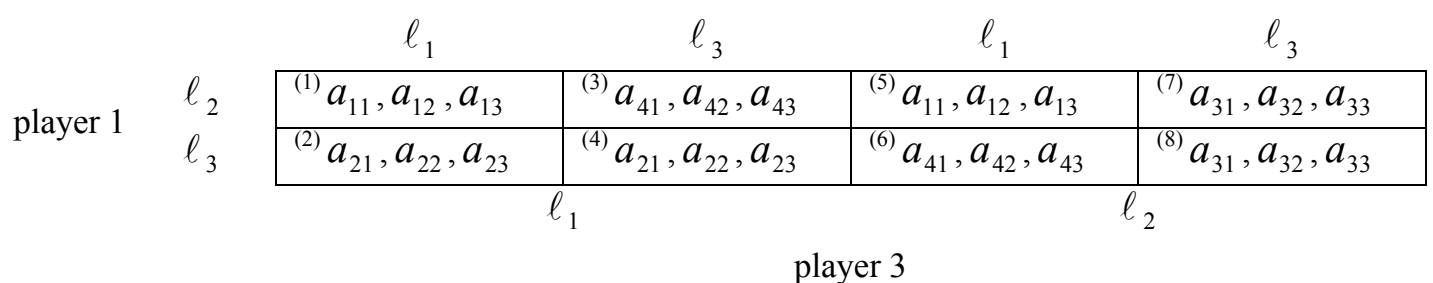

Figure 2. The Normal Form of Three-Player Game (Note 3) 


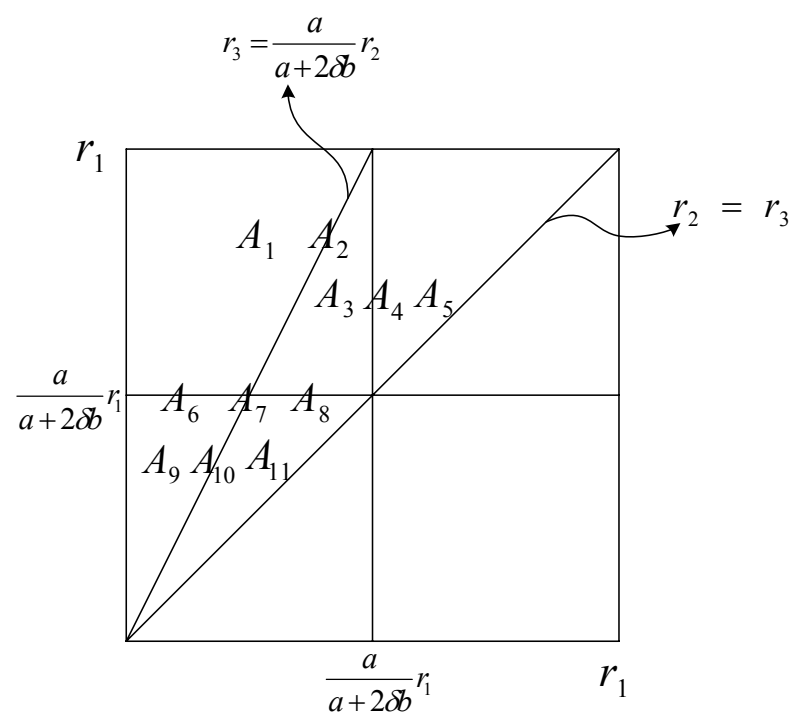

Figure 3. Scope of the Regions

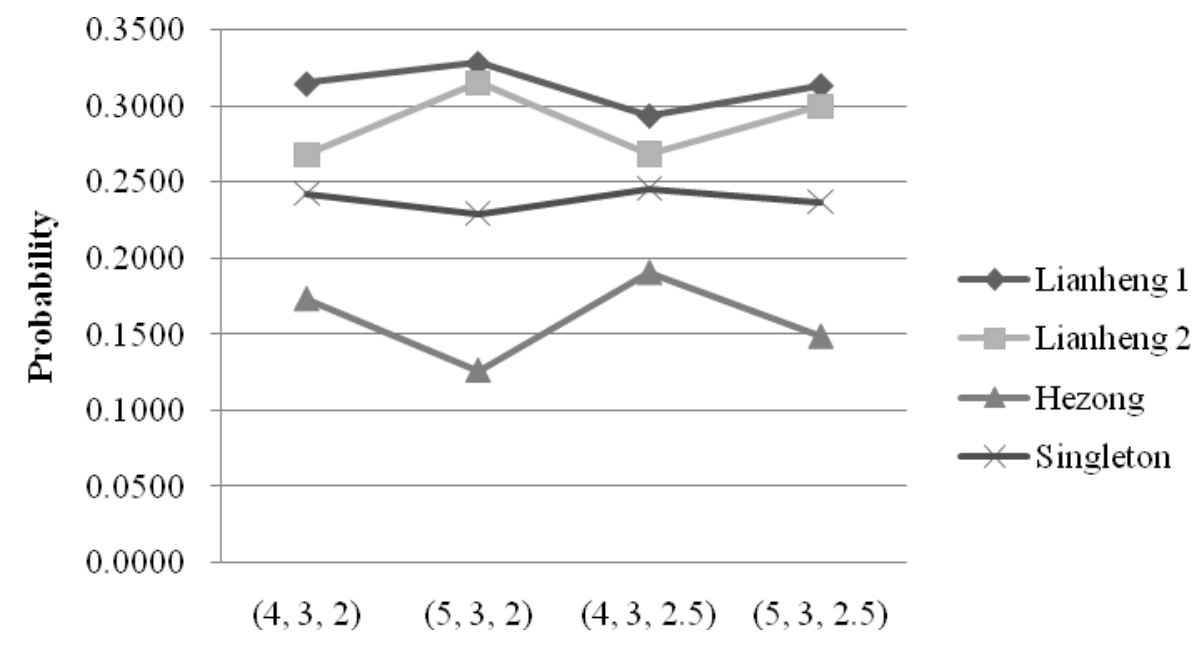

Figure 4. The Probability of Four States when $b=2$ 


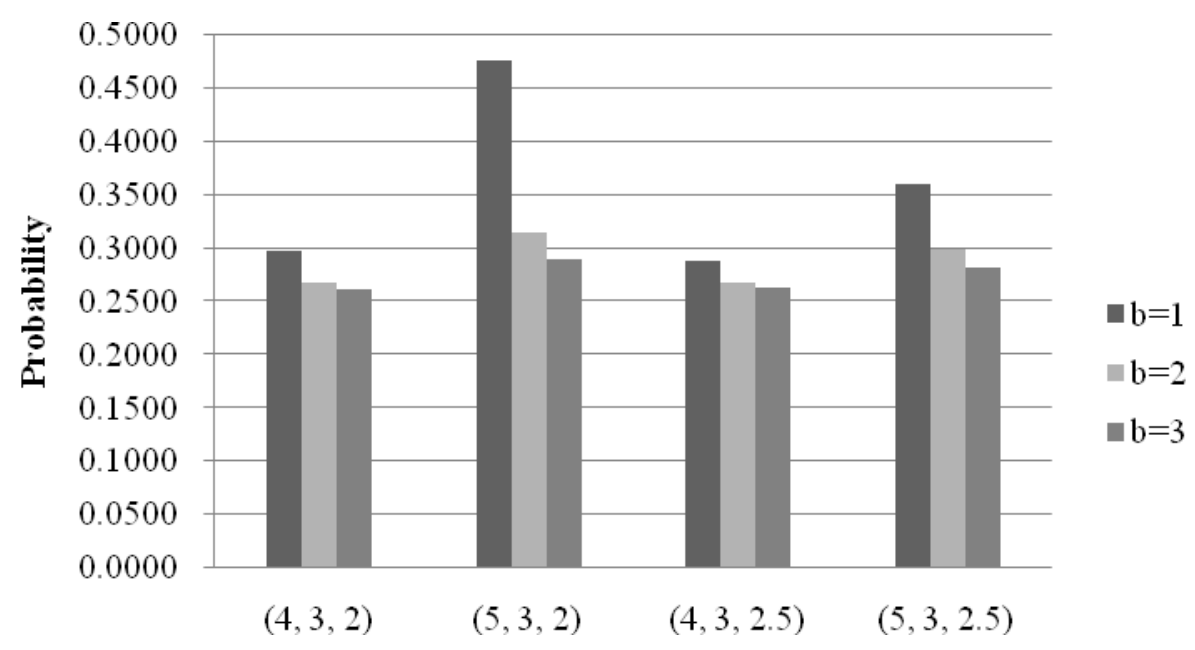

Figure 5. The Probability of Lianheng 2

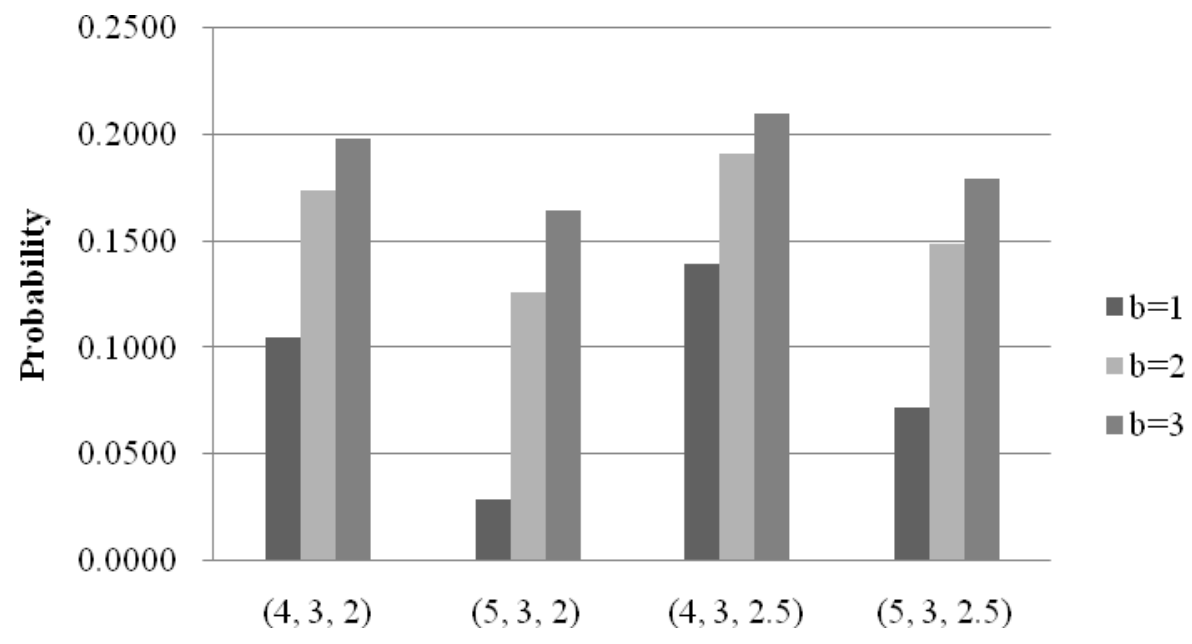

Figure 6. The Probability of Hezong 


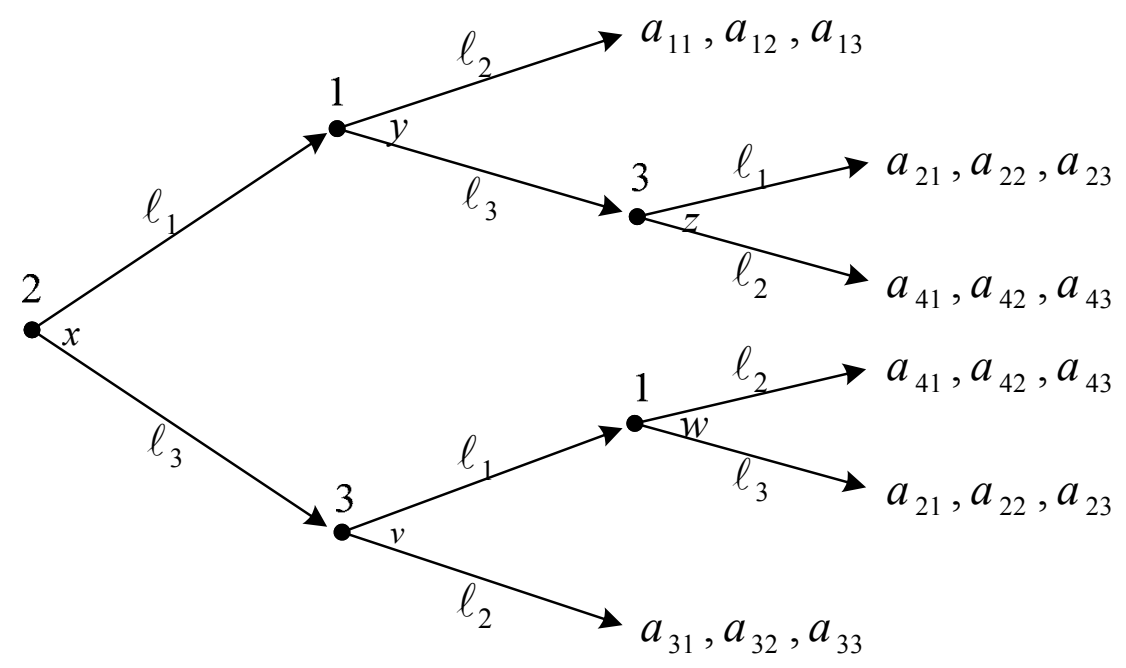

Figure 7. The Extensive Form of Three-Player Game 